\title{
Dynamic QoS Guarantee with Repeater in Power Controlled WCDMA Urban Environment
}

\author{
Mohammad N. Patwary ${ }^{1}$, Predrag Rapajic ${ }^{1}$, Ian Oppermann ${ }^{2}$ \\ ${ }^{1}$ School of Electrical Engineering and Telecommunications, \\ University of New South Wales, Sydney, Australia. \\ ${ }^{2}$ Southern Poro communications \\ 6A, Nelson Street, Annandale, Sydney, Australia
}

\begin{abstract}
In dense urban environment, the possibility of signal reception failed (receiving NAK) repeatedly at the receiving end of the conventional ARQ scheme is nonzero even if with strong FEC as $1 / 3$ turbo code and sometime become noticeable. More robust and modified ARQ protocols are required which may adapt the FEC code rate according to the channel condition. We propose to use repeater as a reliable alternate in this critical channel condition. On the other hand ARQ scheme is efficient only for non-real time communication. Comparing with the ARQ scheme, reliable multipath from repeater will serve with lower time delay, lower SINR requirement and hence more network throughput with the cost of repeaters and multi-user detection scheme (when higher BER performance required).
\end{abstract}

Keywords: Quality of Service (QoS), Line of Sight (LOS).

\section{Introduction}

As the bandwidth of the third generation mobile communications systems increase, radio channel time dispersion can produce noticeably frequency selective fading within the band. The capacity demand on such systems is also increasing, leading to smaller cell size within the network. Multipath scenario has been studied from the very beginning while the CDMA system has been launched. A deterministic approach of multi-path tracking has the advantages of using models of real-network that yields an environment a realistic spread of the signal both in time and angle. Digital transmission using antenna diversity in frequency selective fading channels has been investigated in [1]. The investigations were based on fully decorrelated branch with uncorrelated reflectors with the same average power delay profile at each branch. In [2] the radio channel has been sampled

This work has been supported by Southern Poro Communications, Sydney Australia

The original version of this chapter was revised: The copyright line was incorrect. This has been corrected. The Erratum to this chapter is available at DOI: 10.1007/978-0-387-35618-1_37 
simultaneously in the spatial and temporal domains on two antenna branches and the antenna signal correlation, dispersion and fading for the practical system has been analyzed. A set of antenna diversity for rural macro cell and urban microcell have been shown with array antenna in defuse channel model in [3]. In this paper we analyzed a way to assist with cell layout and to combat interference and fading degradation with optimum label of transmit power and time delay, by using base station diversity using repeater. Our simulation shows that

1. QoS in terms of time delay can be improved significantly by using repeater comparing with the performance of ARQ in Dense urban environment.

2. Using a repeater that provides a strong multipath, there will be $3 \mathrm{~dB}$ gain in BER performance with close loop power control as WCDMA.

We organize the paper in following way. Section 2 describes a System model that has been used for simulation. Section 3 shows the comparison of the time delay performance of the network with repeater and ARQ scheme, section 4 describes the system capacity along with BER performance with and without repeater, section 5 deals with the power control scenario and the network stability and finally the conclusion.

\section{System Model}

For our simulation model we considered a 7-cell cluster, and the central cell serves the test mobile. Mobiles within the network are uniformly distributed and there are regular grids of Base station [4]. To increase the coverage 6 repeaters are placed in every cell in such a way that the coverage radius in the direction of the side-lobe in every sector becomes approximately equal to the coverage radius in the direction of the main lobe radiation. In doing so repeaters two repeaters are placed in the side-lobe radiation edge of every sector of the parent cell (Fig 1). All six repeaters are $60^{\circ}$ apart from each other and $30^{\circ}$ apart from the center of the base station sector main lobe such a way that the signal around the extended radius within the cell is easy to reach. Repeaters are selected such a way that the downlink sensitivity level of the repeater donor antenna is equal to the sensitivity level of user equipment and for uplink repeater antenna sensitivity level is the same as the base station antenna sensitivity level. Repeater uplink amplifier gain is 5dB greater than that of downlink. (These assumptions are made from the repeater specification from the manufacturer). We also consider the terrain is uniform. 


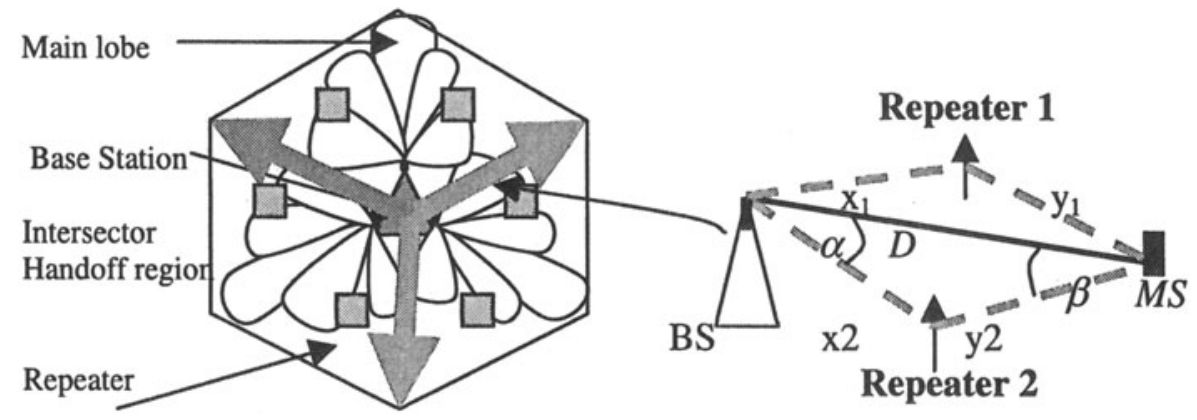

Fig:1- Single cell with three sectors and six Repeaters

Fig. 2: geometrical representation of LOS and Multipath

If the base station antenna height $\mathrm{H}_{\mathrm{bs}}$, the MS antenna height $\mathrm{H}_{\mathrm{ms}}$, and the horizontal distance between the base station and the Mobile station is d, then the effective distance between the BS transmitter and MS receiver is

$D=\sqrt{d^{2}+\left(H_{b s}-H_{m s}\right)^{2}}$

for Urban microcell the difference between the BS and MS antenna height is $15 \mathrm{~m}$ and in rural macro cell $30 \mathrm{~m}$. [4]. If the cell radius $\mathrm{d} \gg\left(\mathrm{H}_{\mathrm{bs}}-\mathrm{H}_{\mathrm{ms}}\right)$ then the effective distance can be considered as the cell radius $d$.

\section{Time delay prediction}

We considered the scenario in a particular sector where there are 2 repeaters been inserted and the repeaters are equidistant from the base station. If the distances between the base station and repeaters are $x_{1} \& x_{2}$, the distances between the repeaters and the test mobile are $y_{1} \& y_{2}$, the angles between the LOS and the multipath to the repeaters are $\alpha_{1} \& \alpha_{2}$ and the angles between the LOS and the received signal path from repeaters to the MS are $\beta_{1} \& \beta_{2}$ respectively then :

$$
\begin{aligned}
& x_{1}=\frac{D \cdot \sin \beta_{1}}{\sin \left[\pi-\left(\alpha_{1}+\beta_{1}\right)\right]} \\
& y_{1}=\frac{D \cdot \sin \alpha_{1}}{\sin \left[\pi-\left(\alpha_{1}+\beta_{1}\right)\right]}
\end{aligned}
$$

$$
\begin{aligned}
& x_{2}=\frac{D \cdot \sin \beta_{2}}{\sin \left[\pi-\left(\alpha_{2}+\beta_{2}\right)\right]} \\
& y_{2}=\frac{D \cdot \sin \alpha_{2}}{\sin \left[\pi-\left(\alpha_{2}+\beta_{2}\right)\right]}
\end{aligned}
$$

Hence the LOS signal will travel a distance $d$ and the multipath signal will travel a distance of $\left(x_{1}+y_{1}\right)$ and $\left(x_{2}+y_{2}\right)$ for their respective repeater. Let the respective time delay between LOS signal and the reflected paths are $\tau_{1}$ and $\tau_{2}$. While the network is designed with the numbers of repeater considered in the system model then the maximum time delay for the receiving process completion will be $\tau_{1}$ and $\tau_{2}$ 


$$
\mathrm{T}=\mathrm{t}+\max \left(\tau_{1}, \tau_{2}\right)
$$

The relation among the $t, \tau_{1}$ and $\tau_{2}$ can be given as follows:

$$
\frac{t}{d}=\frac{t+\tau_{1}}{\left(x_{1}+y_{1}\right)}=\frac{t+\tau_{2}}{\left(x_{2}+y_{2}\right)}
$$

From rectangular geometry it can be found that $\max \left(x_{1}+y_{1}\right)=\max \left(x_{2}+\right.$ $\left.y_{2}\right)=1.4 d$, i.e. transmitted signal can be available to the receiver with sufficient strength only $40 \%$ additional time after the arrival of LOS signal through the repeater. On the other hand, if the signal could not received correctly due to the fading and other attenuation; in particular we are concern about dense urban environment and if there is no available strong reflected path, the conventional solution for this problem is requesting for retransmission of the signal, which is well known as ARQ scheme.

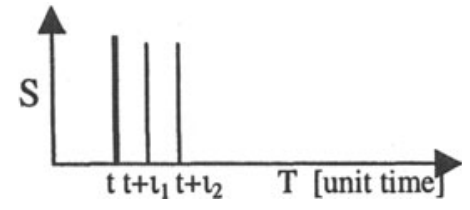

Fig. 3(a): Delay spread scenario with repeater

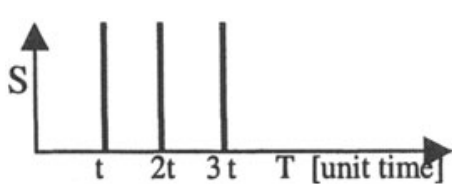

Fig. 3(b): Delay spread scenario with ARQ

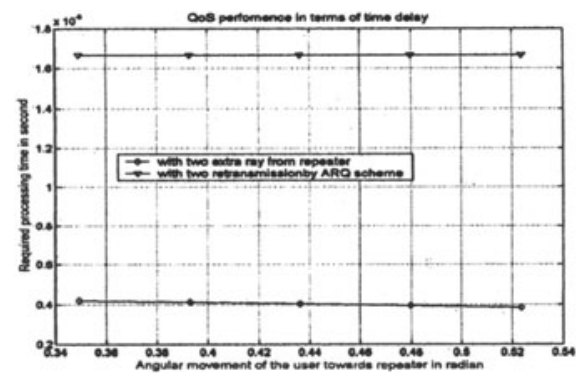

Fig. 4: QoS performance of the network with Repeater and ARQ

Fig 4 shows the test mobile distance vs QoS (in terms of time delay). Comparing with result in [2,5\& 7] we may conclude that for the same amount of BER requirement the system repeater will outperform the system with ARQ scheme and with this delay performance it is possible to serve both real-time and non real-time services.

\section{System Capacity With Repeater}

From the very beginning of the introduction of the repeater for cellular repeater for CDMA [8], to date repeaters are using as a tool to extension of coverage. In urban areas due to the shadowing there is a high probability of deep fading. We for a solution of the shadowing problem is to provide more than one LOS signal to both Uplink and Downlink. In every instant the total 
received power is the vector sum these paths. In the proposed system model it there are two repeaters have been placed in every sector. The carrier to interference plus noise ratio (CINR) of the system for reverse link can be given as

CINR $=\frac{S}{I_{0}}$

Where, $S$ is the Power of test mobile at the BS receive and $I_{0}$ is the total interference and noise. If I represents the total Interference $\mathrm{N}$ represents the total noise (both of them in $\mathrm{dB}$ ) then $\mathrm{I}_{0}$ can be written as follows

$I_{0}=N+I \ldots$ (9) where

$N=k \cdot W \cdot F_{B S} \cdot T$ (Without repeater)

$N=k \cdot W \cdot\left(F_{B S}+F_{\operatorname{Re} p}\right) \cdot T$ (with repeater)...

where $\mathrm{k}$ is boltzman constant, $\mathrm{W}$ is bandwidth, $\mathrm{F}_{\mathrm{BS}}$ and $\mathrm{F}_{\text {rep }}$ stands for base station noise and repeater noise respectively. The interference of the cellular system consists of two components, which are intra-cell interference and inter-cell interference. The measure of these interferences as follows as in [12]

$$
\begin{gathered}
I=\{M(1+\xi)-1\} \cdot V A F \cdot S \ldots(12) \\
S=\frac{P_{m} \cdot G_{B S} \cdot G_{m}}{L} \text { (without repeater) ...(13) } \\
S=\frac{P_{m} \cdot G_{B S} \cdot G_{m} \cdot G_{d} \cdot G_{\text {rep }} \cdot G_{c}}{L \cdot L_{\text {feder }}} \text { (with repeater). }
\end{gathered}
$$

Where, $M, V A F, P_{m}, G, L$ and $\xi$ represents the number of users, Voice activity factor, Power transmit from the mobile, different antenna gain (as suffix), path loss (as suffix, in this analysis we used Hata model) and reuse fraction respectively. Received $\mathrm{E}_{b} / \mathrm{N}_{0}$ can be measured by the following equation

$$
\left(\frac{E_{b}}{N_{0}}\right)_{\text {tot }}=\sum_{i=1}^{M} C I N R_{i} \cdot \frac{W}{R} \cdot V A F
$$

We consider the signal received at the base station receiver is directly from the mobile is of equal strength to the signal from the reflected path through the repeater. The multipath from the repeater has the same delay profile property as from the direct path $[8,9]$ except certain delay whose sustainable limit is maximum $20 \mu \mathrm{s}$ in UMTS (wideband channel)[11] without equalizer. In Fig- 6 it has been shown that with scenario of both signal with equal strength gives the doubled capacity with the repeater than that of the capacity without repeater. Comparing the scenario with the soft hand off it is possible to avoid the performance degradation by using optimal maximal ratio combining diversity instead of selection diversity in forward link and 
the best solution of this problem is adaptive multi-user detection for uplink. The BER of the QPSK modulation can be given by:

$B E R_{Q P S K}=Q\left(\sqrt{\frac{2 E_{b}}{N_{0}}}\right) \ldots$

With different percentage of fading through the channel the system capacity varies in between the dotted and solid line in Fig 5, for every specific bit rate.

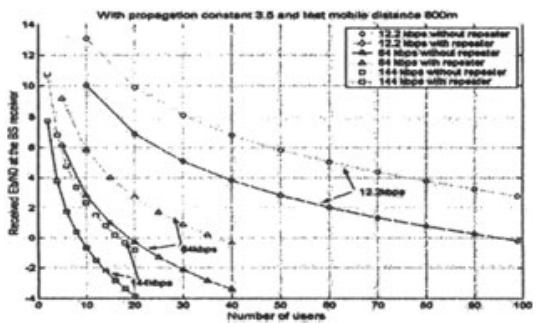

Fig-5 : Number of user vs Received $\mathrm{Eb} / \mathrm{NO}$ with and without repeater for different bit rate.

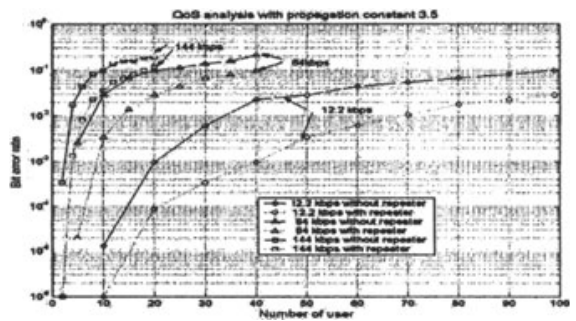

Fig-6: Number of user vs BER curve for different bit rate with and without repeater.

\section{Power Controlled Scenarios}

In our analysis we placed three repeaters per cell to provide an extra multipath with the adaptive directivity by the repeater antenna, where the direct path signal may undergo deep fading due to the shadowing. Each signals suffers from a distance dependent attenuation that can be calculated using the appropriate propagation law.

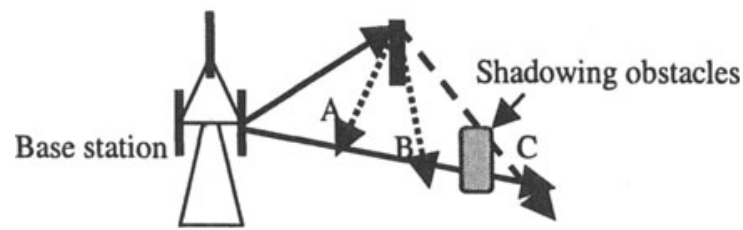

Fig: 7 -showing the schematic signal path from the base station and the repeater to the mobile station. Point A, B and C represent the positions of the mobile.

In Fig 8 Points $A$ and $B$ are outside the shadowing region and $C$ in the shadowing region [6]. For every scenario, total power received by the mobile is the vector sum of all considerable multipath signals. However to demodulated the received signal we used ML multi-user detection. If $S_{1}$ is the signal received through the direct path and $S_{2}$ is the signal received from the reflected path through the repeater then the total instantaneous received power $S_{\text {ins }}$ can be written as

$$
\vec{S}_{\text {ins }}=\vec{S}_{1}+\vec{S}_{2}
$$


If we consider $S_{\text {ins }}$ is the instantaneous required power and $S_{\text {tot }}$ is the total received power, $\mathrm{A}$ factor $\alpha$ introduced as a regulating factor to decide about the transmit power command (TPC) in the closed loop power control technique and the value of $\alpha$ is directly proportional to channel fading.

$$
S_{\text {tot }}=\alpha \cdot S_{\text {ins }} \ldots \text { (18) }
$$

So the controlled received power is then it is always expected to adjust the transmit power to reach the required level according to suitable TPC. It is possible when there is at least one LOS path does exist. Repeater does the same for the shadowed region with the deep fading without degradation of QoS.

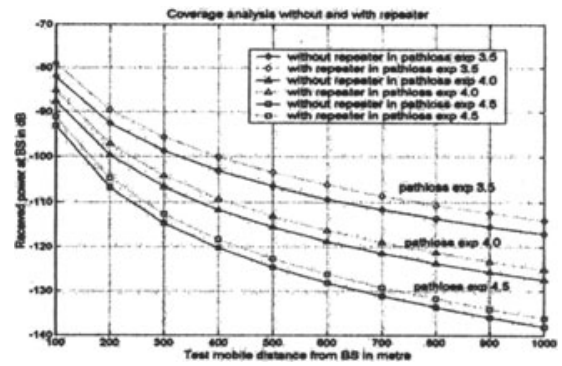

Fig-8: Cell radius vs received power with and without repeaters

In Fig-8 the dotted and the solid line represents the total received power with minimum to maximum value fading in one the multipath channel. It is found that with no fading there is also at least $10 \%$ increment in coverage when repeaters are placed to increase the system capacity. The performances of the repeaters depend on its antennas isolation and the amplifier gain [2,9]. Although the amplifier with the initial stability, the feedback conditions can render the system as a whole unstable which is due the receiving the firing signal from the coverage antenna back to the donor antenna. So as long as any signal leaving the amplifier is attenuated by an amount greater than the amplifier's total gain before it reappears at the amplifier input as feedback or interference, its effect will eventually die away and the system remain stable. However, if the signal attenuation is less than the amplifier gain, results a positive feedback and hence leads to instability [3]. This paper concern about urban areas and we proposed to use repeater to increase capacity only in urban environment. In UMTS closed loop power control tracking which keeps a continuous power monitoring in every $667 \mu \mathrm{s}$, ensures the lower probability of being the network unstable.

\section{CONCLUSION}

In urban area where capacity problem is one of the most common issues that the operators are facing even from the beginning of the cellular system to 
serve the network with least percentage of blocking probability. We proposed to solve this problem by using repeaters within the cellular network to provide an extra multipath (LOS), which not only ensures the minimization of the blocking probability but also increase the instantaneous capacity as well as extension of the coverage. In our future work we are investigating the performance of Adaptive Multi-user detection with repeaters in WCDMA.

\section{REFERENCE}

[1]. B.Glance and L. J. Greenstein, -"Frequency Selective fading effects in digital mobile radio with diversity combining" IEEE Trans. Com vol. 31, no 2, pp 1085 - 11094 Sept. 1983.

[2]. P. C. Eggers, J. Toptgard \& A. M. Opera - “ Antenna system for Base station Diversity in Urban Small and Mocrocell" in IEEE Journal on Selected Area in Communications Vol. 11. No. 7 September 1993 pp 1046 - 1057.

[3]. O. Nфrklit, \& J. B. Anderson - "Defuse Channel Model and Experimental Results for Array Antennas in Mobile Environments" in IEEE Transactions on Antennas and Propagation Vol. 46, No. 6, June 1998 pp. 834 - 840.

[4]. Erceg, D. G. Michelson, S. S. Ghassemzadeh, L. J. Greenstein, A. J. Rustako Jr. P. B. Guerlain, M. K. Dennison, R. S. Roman, D. J. Barnickel, S. C. Wang and R. R. Miller "A Model for the Multipath Delay Profile of Fixed Wireless Channels" - in IEEE Journal on Selected area in Communications vol. 17. No. 3 March 1999, pp 399 410.

[5]. Michele Zorzi -"Some Results on Error Control for Burst-Error Channels Under Delay Constraints" IEEE Transactions On Vehicular Technology, Vol. 50, No. 1, January 2001

[6]. J. P. Decruyenaere and D. Falconer- "A Shadowing Model for prediction of coverage in Fixed Terrestrial Wireless Systems" in VTC 1999 pp 1427-1433

[7]. K. H. Tsioumparakis, T. 1. Doumi and J. G. Gardiner -" Delay spread Statistics in a twopath Radio Environment" in VTC 1996 pp- 642 - 646.

[8] William C. Y. Lee and David J. Y. Lee -" The Impact of repeaters on CDMA System performance" in VTC 2000, pp 1763-1766.

[9]. W. T. Slingsby and J. P. McGeehan - "Antenna isolation Measurements for OnFrequency Repeaters"-IEE Antenna and Propagation conference 4-7 April 1995, pp $239-243$

[10]. R. S. Larkin - "Multiple signal Intermodulation and Stability Considerations in the use of linear repeater" in VTC 1991 pp 747-750.

[11]. K. H. Tsioumparakis, T. 1. Doumi and J. G. Gardiner-"Delay-Spread Considerations of Same Frequency Repeaters in Wideband Channels"- in IEEE Trans. on Vehicular Technology Vol. 46, No 3, August 1997, pp $664-675$.

[12]. J. S. Lee and L. E. Miller- " CDMA System Engineering Handbook"- Artech House Publishers. 